\title{
INFORMATIONS GENERALES
}

\author{
Pré Programme
}

\section{4ème Congrès de la}

\section{Société d'Andrologie de Langue Française}

\section{TOULOUSE \\ 11-13 décembre 1997}

ATRIA TOULOUSE CENTRE

8, ESPLANADE CONFANS CAFFARELLI

Renseignements -

R. MIEUSSET - Centre de Stérilité Masculine Hôpital La Grave 31052 TOULOUSE CEDEX ₹ : 0561777901 - Télécopie : 0561777843

Email : mieusset @ cict.fr 
$8 \mathrm{H} 30$ : Accueil

\section{H00 CONFERENCE D'ACTUALITE}

Baisse de la production et de la qualité spermatique chez l'homme ? Facteurs de variation et problèmes méthodologiques.

J. AUGER (F)

10H00 Pause Café

$10 H 30$ CONGELATION DES GAMETES MALES

- Etat de la question

G. GRIZARD (F)

- Techniques de congélation.

J. CRISTER (USA)

- Congélation de tissu testiculaire: indications, techniques.

A. CLAVERT

- Qualité des gamètes après congélation/décongélation

S. HAMAMAH (F)

12H30: Déjeuner

$14 \mathrm{HOO}$

\section{SYMPOSIUM}

ANDROGENES ET FOLLICULE PILEUX

Avec la collaboration des Laboratoires MSD-CHIBRET

- Introduction

Ch. SULTAN (F)

- Les 5 alpha-réductases : découverte et application en pathologie humaine

L. RHODES (USA)

- Distribution des isotypes de la 5 alpha-réductase dans le follicule pileux

E. BAYNE (USA)

- Mécanisme d'action du finastéride sur la 5 alpha-réductase G. HARRIS (USA)

- Résultats des études de phase III du finastéride dans le traitement de l'alopécie androgénétique

Ph. SAIAG (F)

16H00-17H00: $\quad$ Visite des POSTERS et Pause Café

\section{H00-18H30 ROLE DES OESTROGENES CHEZ LE MALE}

- L'aromatase testiculaire: du gène à la protéine

S. CARREAU (F)

- Résistance aux oestrogènes: un modéle d'étude des actions des oestrogènes chez le mâle

Ch. SULTAN (F)

- Analyse de l'action des oestrogènes chez le mâle au moyen de souris transgèniques

K.S. KORACH (USA)

\section{FORMATION/ MISE AU POINT}

20H30 Genetique moleculaire de l'axe gonadotrope.

21H30 Identification des cellules rondes du sperme.

Ph. BOUCHARD (F)

E. VENDRELY (F) 


\section{$8 H 15$ CONFERENCE D'ACTUALITE}

Apport de l'echographie dans l'exploration de l'infecondite masculine

E. SCHOUMAN-CLAEYS (F)

\section{H00 TRAITEMENTS DES HOMMES AYANT UNE OAT "IDIOPATHIQUE"}

- Diagnostic, traitement et pronostic des obstructions "partielles" des voies excretrices L. SEGAL (B)

- Les risques cytogénétiques pour le zygote en rapport avec les techniques récentes d'A.M.P. L. KOULISCHER (B)

- Traitements médicaux des OAT idiopathiques. F. COMHAIRE (B)

- Méta-analyse des traitements de l'OAT idiopathique et synthèse. A. DEMOULIN (B)

11H00-12H15 : $\quad$ Visite des POSTERS et Pause Café

$12 H 30$ : Déjeuner

14H00 SEXUALITE : ETHIQUE, RELIGION, CULTURE ET SOCIETE.

Avec, entre autres, la participation de Robert Misrahi, philosophe, Thahar Ben Jelloun, écrivain

17H00 : Pause Café

17H30: PRESENTATION des POSTERS SELECTIONNES REMISE des PRIX POSTERS

18H30-19H30 :

\section{ASSEMBLEE GENERALE SALF FORMATION/ MISE AU POINT}

20H30 Place des tests d'interaction avec le mucus dans le bilan d'infécondité

21H30 Immunité anti-spermatozoïdes et AMP

Y. SOFFER (Is)

M. DE ALMEIDA (F) 
SAMEDI 13 DECEMBRE 1997

\section{H30 CONFERENCE D'ACTUALITE}

Où en est standardisation de l'exploration de l'impuissance en 1997?

J. BUVAT (F)

9H0O EJACULATION PREMATUREE

- Epidémiologie de l'éjaculation prématurée

A. BEJIN (F)

- De la physiologie à la clinique

F. GIULIANO (F)

- Définitions à l'épreuve de la clinique

M. CHEVRET-MEASSON (F)

\section{$10 \mathrm{H30}$ Pause Café}

\section{$11 \mathrm{HO0}$ SYMPOSIUM}

RESPONSABILITE MEDICALE EN ANDROLOGIE

avec le concours du Sou Médical

et le patronage des Laboratoires Synthélabo

$12 H 30$ Déjeuner de cloture

POSTERS

- sur une page sous la forme : titre, auteurs et affiliation, introduction, matériel et méthodes, résultats, discussion

- à adresser avant le 01 Novembre 1997 à

Dr E. Szerman Laboratoire de Biochimie, CHU

av. de la Côte de Nacre 14033 Caen Cedex.

\section{INSCRIPTIONS}

- Pour l'ensemble du Congrès (repas midi inclus) :

Etudiants, internes, para-médicaux

avant $1 / 11 / 97$

Membres de la SALF

$600 \mathrm{~F}$

$1000 \mathrm{~F}$

après

Non membres de la SALF

$1400 \mathrm{~F}$

$600 \mathrm{~F}$

$1200 \mathrm{~F}$

$1600 \mathrm{~F}$

- A la journée (repas midi inclus) : $\quad 400 \mathrm{~F}$ 


\title{
Programme préliminaire \\ Traitements anticancéreux et fertilité Protection de la fonction de reproduction
}

\author{
Ecole Normale Supérieure de LYON \\ 9 JANVIER 1998
}

\author{
Coordinateurs : J.F.GUERIN et L.P.NACHURY \\ avec le soutien scientifique du réseau INSERM «Pro-sperm»
}

Renseignements : Pr J.F. GUERIN - Laboratoire de Biologie de la Reproduction

8, Av. Rockefeller - 69373 LYON CEDEX 08

Tél. : 04-78-77-70-64 ; Fax : 04-78-77-72-64

\section{Allocution d'ouverture}

Effet des traitements anticancéreux sur la fonction de reproduction

Modérateurs : T. PHILIP, L.P.NACHURY

- Une préoccupation née des progrès conjoints de la cancérologie et de la biologie T. PHILIP

- Position du problème : - chez l'adulte (J.D. TIGAUD)

- chez l'enfant (G. SOULLIET)

Données épidémiologiques Christine LASSET $15 \mathrm{~min}$

-Incidence des Cancers dans la descendance des patients cancéreux D.FRAPPAZ 15 min

-Impact des chimiothérapies sur la gamétogenèse J.P.DROZ 15 min

-Impact des radiothérapies P. ROMESTAING 15 min

-Conséquences de la chirurgie sur la fonction de reproduction masculine M.DEVONEC $10 \mathrm{~min}$

Pause-café $20 \mathrm{~min}$

-Effets des traitements anticancéreux sur le patrimoine génétique de la descendance .

D.MARMOR 15 min

-Discussion générale

Moyens actuels de protection de la fonction de reproduction, et perspectives

- Modérateurs : J.F.GUERIN et H.LEJEUNE

-Cadre juridique : la loi de Bioéthique J.C. CZYBA 10 min

\section{PAUSE DEJEUNER}

-Conservation du sperme, des ovocytes et des embryons D. COTTINET

-Nouvelles techniques d'Assistance Médicale à la Procréation (ICSI) J.F. GUERIN

-Protection de la spermatogenèse chez l'animal et chez l'homme B. JEGOU $20 \mathrm{~min}$

-Conservation de tissu testiculaire et maturation in vitro de la lignée germinale H. LEJEUNE $15 \mathrm{~min}$

-Protection de la fonction ovarienne L.P.NACHURY $15 \mathrm{~min}$

-Conservation de tissus ovariens :

-Aspects cryobiologiques et chirurgicaux Y.AUBARD / B. SALLE $15 \mathrm{~min}$

-Maturation de follicules in vitro J. LORNAGE $10 \mathrm{~min}$

\section{Pause-café}

-Aspects psychologiques : du diagnostic de cancer au désir d'enfant P. SALTEL 15 min ; Que représente l'autoconservation du sperme pour un homme atteint d'un cancer ? J.L. CLÉMENT, $10 \mathrm{mn}$.

Synthèse : Table ronde faisant intervenir : biologistes de la reproduction, gynécologues, pédiatres, cancérologues (orateurs pressentis : Mme Brunat-Mentigny, Mrs A. Brémond, J.C. Czyba, D. Dargent, J.P. Gérard, T. Philip, N. Philippe, H. Plauchu, J.M. Thoulon). 


\title{
International Symposium on MALE STERILITY FOR MOTILITY DISORDERS : ETIOLOGICAL FACTORS AND TREATMENT (Serono Symposia)
}

" ASIEM », 6, rue Albert de Lapparent, 75007 Paris

30-31 Janvier 1998

\begin{abstract}
Vendredi 30 Janvier
Session I - Immotile sperm : etiology

8h30 Mechanism and regulation of sperm motility : an update J.L. GATTI (France)

9h Metabolic strategy in human spermatozoa : its impact on sperm motility J. G.ALVAREZ (USA)

9h30 Extrinsic factors affecting sperm motility : immunological, infectious, toxic factors and ROS Cl. GAGNON (Canada)

10h00 Intracellular Ca 2 + and sperm motility H. BREITBART (Israel)

11h00 Genetic aspects of sperm motility disorders J. CUMMINS (Australia)

11h30 Necrozoospermia : etiology and management P. LECOMTE (France)

12h00 Presentation of selected posters
\end{abstract}

\section{Session II - Therapeutic possibilities}

14h00 Clinical assessment of flagellar dyskinesia P. JOUANNET, J.P. WOLFF, J. AUGER (France)

14h20 Medical treatments R. MIEUSSET (France)

$14 \mathrm{~h} 40$ In vitro therapy of sperm motility : specific and non specific treatment S. HAMAMAH (France)

\section{Session III - ICSI option for immotile sperm}

15h20 Identification of live immotile sperm : HOST, supravital staining J. COHEN (USA)

$15 \mathrm{~h} 40$ Injection procedure of immotile sperm : selection, tail breakage, PVP, medium culture S. FISHEL (U.K)

16h00 Fertilizing capability of thawed immotile sperm R. RON-EL (Israel)

$16 \mathrm{~h} 20$ Clinical aspects of ICSI with immotile sperm H. TOURNAYE (Belgium)

16h40 ICSI IN DIFFICULT CASES : results of short report H. TOURNAYE (Belgium), J.F.GUERIN (France), M. ABOULGAR(Egypt), Y. SOFFER (Israel), S. FISHEL (U.K), J. COHEN (U.S.A) 


\section{Samedi 31 Janvier}

Session IV - Testicular sperm : physiological to pathological aspects

8h30 Growth factors in the testis developpement and function : an update M. BENHAMED (France)

9h00 Immature sperm cells : molecular mechanism of fertilization : an update A. OGURA, R. YANAGIMACHI (Japan, USA)

9h30 Testicular apoptosis L.D. RUSSEL (USA)

10h00 Expression of genes involved in the regulation of apoptosis in the testis D.DE ROOIJ (Netherlands)

\section{Session V - Testicular sperm retrieval}

11h00 Microcaptors interest for testicular sperm extraction before to refer to ICSI G. TRITTO, A. DITMAR, G. ARVIS (France)

11h30 Sperm testicular extraction : Methods, modalities and ideal sites for sperm retrieval S. SILBERT (USA)

12h00 Possible pathological risks following multiple biopsies for TESE ? P.N. SCHELGEL (USA)

\section{Session VI - Conceptus quality in ICSI with testicular sperm}

14h30 Genetic aspects of male sterility P.H. VOGT (Germany)

$15 \mathrm{~h} 00$ Conceptus quality from ICSI with testicular sperm R. FRYDMAN, F. OLIVENNES (France)

15h30 Genetic problems of ICSI I. LIEBAERS, M. BOUNDUELLE (Belgium)

16h30 Ethical problems of assisted conception F. SHENFIELD (U.K.)

\section{Secrétariat Scientifique}

S.HAMAMAH, Unité de Biologie de la Reproduction

Dépt. de Gynécologie Obstétrique

Hôpital Bretonneau, 37044 Tours

Tél. 33-2-47-47-47-46

Fax : 33-2-47-61-08-06

E.mail : hamamah @ med.univ-tours.fr

\section{Information}

Maria Grazia CALI

Director, Serono-Symposia SA

P.O. Box 7228

00100 Roma (Nomentano), Italie

Tél. 39-6-70-38-45-06

Fax : 39-6-70-38-45-77 